\title{
Calculation of the crystal-melt interfacial free energy of succinonitrile from molecular simulation
}

\author{
Xiaobing Feng and Brian B. Laird ${ }^{\text {a) }}$ \\ Department of Chemistry, University of Kansas, Lawrence, Kansas 66045
}

(Received 24 October 2005; accepted 14 November 2005; published online 30 January 2006)

\begin{abstract}
The crystal-metal interfacial free energy for a six-site model of succinonitrile $\left[\mathrm{N} \equiv \mathrm{C}-\left(\mathrm{CH}_{2}\right)_{2}-\mathrm{C}\right.$ $\equiv \mathrm{N}]$ has been calculated using molecular-dynamics simulation from the power spectrum of capillary fluctuations in interface position. The orientationally averaged magnitude of the interfacial free energy is determined to be $(7.0 \pm 0.4) \times 10^{-3} \mathrm{~J} \mathrm{~m}^{-2}$. This value is in agreement (within the error bars) with the experimental value $\left[(7.9 \pm 0.8) \times 10^{-3} \mathrm{~J} \mathrm{~m}^{-2}\right.$ ] of Maraşli et al. [J. Cryst. Growth 247, 613 (2003)], but is about $20 \%$ lower than the earlier experimental value $\left[(8.9 \pm 0.5) \times 10^{-3} \mathrm{~J} \mathrm{~m}^{-2}\right]$ obtained by Schaefer et al. [Philos. Mag. 32, 725 (1975)]. In agreement with the experiment, the calculated anisotropy of the interfacial free energy of this body-centered-cubic material is small. In addition, the Turnbull coefficient from our simulation is also in agreement with the experiment. This work demonstrates that the capillary fluctuation method of Hoyt et al. [Phys. Rev. Lett. 86, 5530 (2001)] can be successfully applied to determine the crystal-melt interfacial free energy of molecular materials. ㅇ 2006 American Institute of Physics. [DOI: 10.1063/1.2149859]
\end{abstract}

\section{INTRODUCTION}

The crystal-melt interfacial free energy, $\gamma$, is defined as the reversible work required to create an interface of unit area between a crystal and its coexisting melt. Both the magnitude and anisotropy (orientation dependence) of $\gamma$ are crucial parameters in determining the kinetics and morphology of crystal nucleation and crystal growth. ${ }^{1,2}$ For example, the anisotropy of $\gamma$ has a profound effect on dendritic growth, even small anisotropies on the order of $1 \%-4 \%$, which are typical in metals, are significant in determining dendrite shapes. ${ }^{3,4}$ In addition, the nucleation rates of crystals ${ }^{5,6}$ and colloids $^{7}$ exhibit a strong dependence on $\gamma$. Direct methods to measure $\gamma$ experimentally are difficult and have been applied to relatively few materials. ${ }^{8}$ Such direct experiments often involve contact angle measurements and are often not of sufficient precision to determine the anisotropy in $\gamma$. However, the anisotropy of $\gamma$ has been measured directly for a small number of transparent materials, such as succinonitrile and pivalic acid, ${ }^{9,10}$ water, ${ }^{11}$ ammonium bromide, ${ }^{12}$ and hexaoctyloxytriphenylene. ${ }^{13}$ For the majority of materials, the value of the interfacial free energy is extracted indirectly from nucleation data. ${ }^{1,14,15}$ Such indirect measurements are only accurate to $10 \%-20 \%$, on average, due to the approximations inherent in classical nucleation theory, which is used to extract $\gamma$ from nucleation rates. In addition, values so obtained represent averages over orientation, so all information as to anisotropy is lost. The difficulty in obtaining reliable experimental measurements has motivated the recent development of two complementary methods to determine $\gamma$ via molecular simulation-the cleaving method ${ }^{16,17}$ and the fluctuation method. ${ }^{18}$ These methods have had success in determining both the magnitude and anisotropy of $\gamma$ for simple

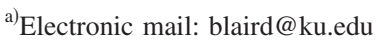

systems, such as hard spheres, ${ }^{17,19}$ inverse-power potentials ${ }^{19}$ and Lennard-Jones particles, ${ }^{16,20,21}$ as well as for metals ${ }^{18,22,23}$ and metal alloys. ${ }^{24}$ In this work, we apply the fluctuation method to calculate the interfacial free energy for a molecular system, namely, the organic compound succinonitrile.

In recent years, succinonitrile $(\mathrm{SCN})$

$$
\mathrm{N} \equiv \mathrm{C}-\left(\mathrm{CH}_{2}\right)_{2}-\mathrm{C} \equiv \mathrm{N}
$$

has become a material of interest in the study of solidification kinetics. SCN, like many metals of technological interest (for example, $\mathrm{Fe}$ ), freezes into a body-centered-cubic (bcc) crystal structure. ${ }^{25}$ Unlike face-centered-cubic (fcc) metals, however, SCN is low melting $\left(T_{m}=331 \mathrm{~K}\right)$ and transparent, making it far easier to study experimentally. As a result, SCN is used as a model material for the study of the solidification kinetics of bcc-forming systems. Also, the succinonitrile-acetone alloy is used as a model material for the study of crystal growth and solidification of metal alloy systems. ${ }^{26,27}$ The importance of SCN in solidification studies has been underscored recently by its use in a series of experiments of bcc dendritic growth kinetics in microgravity. ${ }^{28}$

The primary purpose of the current work is to determine whether the interfacial free energy of a molecular material can be accurately calculated by computer simulation. We have chosen SCN as a test candidate because experimental values for both the magnitude and the anisotropy of the interfacial free energy exist for comparison. ${ }^{9,10}$ The first measurement of SCN crystal-melt interfacial free energy by Schaefer et al. ${ }^{29}$ using a grain-boundary angle method, produced an orientationally averaged value of $(8.9 \pm 0.5)$ $\times 10^{-3} \mathrm{~J} \mathrm{~m}^{-2}$. A recent measurement by Maraşli et al. ${ }^{30}$ using a similar technique, obtained a somewhat lower value of $(7.9 \pm 0.8) \times 10^{-3} \mathrm{~J} \mathrm{~m}^{-2}$. In the same paper, Maraşli et al. used their numerical model to reexamine the data of Schaefer 
et al., ${ }^{29}$ obtaining a value of $8.02 \times 10^{-3} \mathrm{~J} \mathrm{~m}^{-2}$. In the experiments, the orientation dependence of $\gamma$ for a crystal with cubic symmetry is often represented to low order by the expansion,

$$
\gamma(\theta)=\gamma_{1}\left[1+\epsilon_{4} \cos (4 \theta)\right],
$$

where $\gamma_{1}=\left(\gamma_{100}+\gamma_{110}\right) / 2$, the average of the interfacial free energies of (100) and (110) interfaces. $\epsilon_{4}$ is an anisotropy parameter, and $\theta$ the angle between the interface normal and the [100] direction in the crystal. The anisotropy parameter $\epsilon_{4}$ was measured by both Glicksman and Singh ${ }^{9}$ and Muschol et al. ${ }^{10}$ to be $0.5 \%$ and $(0.55 \pm 0.15) \%$, respectively. This anisotropy is an order of magnitude smaller than that of pivalic acid, a model material for the study of the solidification of fcc materials, for which anisotropies of 5\% (Ref. 9) and $(2.5 \pm 0.2) \%$ (Ref. 10) have been measured in separate experiments. The lower value of the anisotropy for bcc materials compared with that of fcc materials appears to be a general phenomenon and has been also observed in simulations of soft repulsive spheres ${ }^{19}$ and of various model potentials for iron. ${ }^{23}$

\section{MEASURING $\gamma$ IN MOLECULAR SIMULATION}

Two qualitatively different, but complementary, simulation methods have been proposed for the calculation of $\gamma$ and its anisotropy. This first technique, the cleaving method, ${ }^{16,17,19,20}$ uses external potentials to construct a reversible path between an initial state consisting of separated bulk crystal and melt and a final state in which the crystal and melt coexist in equilibrium separated by an interface. The interfacial free energy is then directly determined for any crystal orientation as the reversible work along constructed path. (For a more complete review of both the cleaving and fluctuation methods see Ref. 31.)

In the fluctuation method, ${ }^{18,21-24,32}$ which is used in this work, the interfacial free energy is determined from the power spectrum of the fluctuations of the interfacial position. In this technique, a slab geometry is used for the simulation box, in which the longest direction is perpendicular to the average interfacial plane. Of the two directions parallel to the interfacial plane, one (defining the width $W$ ) is about onehalf the length of the longest direction, and the other (defining the thickness $b$ ) is only a few atomic layers in length, which makes the interfacial position $h(x)$ a quasi-onedimensional function of the distance along the width of the box. According to the capillary fluctuation method the equilibrium fluctuation spectrum of the crystal-melt interface height of a quasi-one-dimensional interface can be expressed as $^{18}$

$$
\left\langle|\tilde{h}(k)|^{2}\right\rangle=\frac{k_{B} T}{b W \widetilde{\gamma} k^{2}},
$$

where $k_{B}$ and $T$ are Boltzmann's constant and the melting temperature, respectively. The function $\tilde{h}(k)$ is the onedimensional Fourier transform of $h(x)$ at wave number $k$, and $b$ and $W$ are as defined above. In this expression the angular brackets denote ensemble average. The quantity $\tilde{\gamma}$ is called the interfacial stiffness and is related to the interfacial free energy by

$$
\widetilde{\gamma}=\gamma+\frac{d^{2} \gamma}{d \theta^{2}},
$$

where $\theta$ is the angle between the instantaneous interface normal and the average interface normal. Using Eq. (2), a plot of $\ln \left\langle|\tilde{h}(k)|^{2}\right\rangle$ versus $\ln (k)$ should be linear with a slope of -2 . The value of the interfacial stiffness can be determined from the intercept of this plot. Once $\tilde{\gamma}$ has been measured for several interfacial orientations, the value of $\gamma$ in each orientation is obtained indirectly by constructing a functional form for the dependence of $\gamma$ on orientation and fitting $\tilde{\gamma}$ to obtain the best fits for the parameters of the functional form. One such expansion, ${ }^{33}$ recently applied in a number of studies, ${ }^{20,21,24}$ is

$$
\begin{aligned}
\gamma(\hat{\mathbf{n}}) / \gamma_{0}= & 1+\epsilon_{1}\left(\sum_{i=1}^{3} n_{i}^{4}-\frac{3}{5}\right) \\
& +\epsilon_{2}\left(3 \sum_{i=1}^{3} n_{i}^{4}+66 n_{1}^{2} n_{2}^{2} n_{3}^{2}-\frac{17}{7}\right),
\end{aligned}
$$

where $\left\{n_{1}, n_{2}, n_{3}\right\}$ are the Cartesian components of the interface normal $\hat{\mathbf{n}}, \gamma_{0}$ is the orientationally averaged interfacial free energy, and $\epsilon_{1}$ and $\epsilon_{2}$ are expansion coefficients that quantify the anisotropy. Because the interfacial stiffness is an order of magnitude more anisotropic than $\gamma$ itself, ${ }^{18}$ the interface fluctuation method, which directly measures $\gamma+\gamma^{\prime \prime}$, is very sensitive to small anisotropies in $\gamma$. This method thus gives very precise values of the anisotropy values $\left(\epsilon_{1}\right.$ and $\left.\epsilon_{2}\right)$ in Eq. (4). However, because $\gamma$ itself is not measured directly, but instead is indirectly fitted to the stiffness data using Eq. (4), the precision in the magnitude of $\gamma$ is lowered.

The two methods can be viewed as complementary, in that the cleaving method gives a more precise value for the magnitude of $\gamma$ (because $\gamma$ is measured directly), whereas the anisotropy of $\gamma$ is better resolved by the fluctuation method. The two methods have been shown to produce identical results (within numerical uncertainty) for a truncated LennardJones system. ${ }^{20,21}$ Both methods indicate that $\gamma_{100}>\gamma_{110}$ $>\gamma_{111}$ for all fcc materials studied. In addition, both methods have also shown that bcc systems have smaller magnitude and anisotropy in interfacial free energy than for fcc systems. ${ }^{19,23}$ These studies also have shown that the amplitude and anisotropy of the interfacial free energy is sensitive to the potential. ${ }^{19,23}$ For bcc soft spheres, the relationship $\gamma_{100}>\gamma_{111}>\gamma_{110}$ is found with the cleaving method. ${ }^{19}$ The calculation on Fe with the fluctuation method gives different ordering for different potentials of Fe.

It is our eventual goal to determine the interfacial free energy of succinonitrile using both the cleaving and fluctuation techniques. We have chosen the fluctuation method for this first study because there remain some technical issues with respect to optimal manner in which to construct cleaving walls for systems with Coulomb interactions. 
TABLE I. The interface orientations and system sizes. The notation of Ref. 21 is used to label interface orientation, that is, the square brackets denote the "short" direction of the simulation box. The lattice constant of plastic SCN is $6.4095 \AA$ at $332 \mathrm{~K}$, which is the simulation temperature.

\begin{tabular}{ccc}
\hline \hline Orientation & Size $($ in $\AA)$ & Molecules \\
\hline$(100)[001]$ & $25.64 \times 192.28 \times 388.37$ & 14400 \\
$(110)[001]$ & $25.64 \times 182.28 \times 366.16$ & 12800 \\
$(111)[1 \overline{1} 0]$ & $27.19 \times 157.00 \times 403.61$ & 12960 \\
\hline \hline
\end{tabular}

\section{THE INTERFACIAL FREE ENERGY OF SUCCINONITRILE}

Before beginning simulations on succinonitrile, it is necessary to define a molecular interaction potential. We use a recently developed six-site force field for SCN. ${ }^{34}$ This force field was chosen because it gives a melting temperature $(332 \mathrm{~K}$ at $1 \mathrm{bar})$ that is very close to the experiment $(331 \mathrm{~K})$ and it has been shown that the interfacial free energy for fcc metals is strongly correlated with the melting temperature. ${ }^{35}$ This six-site interaction potential was shown to produce a more well-defined crystalline state and better melting point than an earlier four-site force field. ${ }^{36}$ In addition, the proportion of trans conformers $(20 \%)$ in the crystalline state is in good agreement with the experiment $(23 \%){ }^{37}$

The molecular-dynamics (MD) simulations were performed using the DLPOLY package ${ }^{38}$ at constant $N, V$, and $T$ using a Berendsen thermostat to hold the temperature constant at the melting temperature $(332 \mathrm{~K})$. A 1 fs time step was used in all simulations. To create the equilibrium interface we use the following procedure.

- Slab-shaped crystal and melt samples were prepared separately.

- The crystal and melt slabs were joined together to form an interface. A gap is created at the interface to avoid overlapping, and the melt was scaled to maintain the correct density.

- Solid atoms were held fixed and melt molecules were allowed to fill the gap and come to equilibrium in contact with the frozen crystal. The total time for this step in each run was between 60 and 80 ps.

- The entire system was equilibrated for 50-60 ps.

- To generate data for analysis a total of between 3800 and 4200 system configurations were stored. The interval between each stored configuration was $100 \mathrm{fs}$.

A minimum of three interface orientations are needed to extract $\epsilon_{1}, \epsilon_{2}$, and $\gamma_{0}$ using Eq. (4). The orientations and system sizes used in this work are summarized in Table I. The accuracy of the fitting process is improved by using more than the minimum number of three orientations; however, because of the large number of molecules necessary in these simulations (14000 molecules $=84000$ atomic interaction sites) we were restricted to only three orientations for the present calculation. For the slab geometry used in the fluctuation model, both the interface normal and the "short" direction must be specified for a given orientation. The three box orientations used here are (100)[001], (110)[001], and (111)[1 $\overline{1} 0]$ where the numbers in parentheses are Miller indices for the interface normal and the numbers in square brackets are those for the short direction of the simulation box. In terms of the Fehlner-Vosko coefficients, the stiffnesses for these three orientations are

$$
\begin{aligned}
& \tilde{\gamma}_{(100)[001]}=\gamma_{0}\left(1-\frac{18}{5} \epsilon_{1}-\frac{80}{7} \epsilon_{2}\right), \\
& \tilde{\gamma}_{(110)[001]}=\gamma_{0}\left(1+\frac{39}{10} \epsilon_{1}+\frac{155}{14} \epsilon_{2}\right), \\
& \tilde{\gamma}_{(111)[110]}=\gamma_{0}\left(1+\frac{12}{5} \epsilon_{1}-\frac{1280}{63} \epsilon_{2}\right) .
\end{aligned}
$$

The simulations are analyzed using Eq. (2) to obtain the raw stiffness values, which are used to obtain $\epsilon_{1}, \epsilon_{2}$, and $\gamma_{0}$. The free energies for the (100), (110), and (111) interfaces can be obtained,

$$
\begin{aligned}
& \gamma_{100}=\gamma_{0}\left(1+\frac{2}{5} \epsilon_{1}+\frac{4}{7} \epsilon_{2}\right), \\
& \gamma_{110}=\gamma_{0}\left(1-\frac{1}{10} \epsilon_{1}-\frac{13}{14} \epsilon_{2}\right), \\
& \gamma_{111}=\gamma_{0}\left(1-\frac{4}{15} \epsilon_{1}+\frac{64}{63} \epsilon_{2}\right) .
\end{aligned}
$$

In order to determine the interface height $h(x)$, it is necessary to distinguish between "crystal" and "melt" atoms. To do this, we define an order parameter for a molecule of the form, ${ }^{22}$

$$
\phi=\left|\frac{1}{N_{q} Z} \sum_{\mathbf{r}, \mathbf{q}} e^{i \mathbf{q} \cdot \mathbf{r}}\right|^{2},
$$

where $Z$ is the number of nearest neighbors of the molecule and $N_{q}$ the number of reciprocal vectors $\mathbf{q}$ that satisfy $\exp (i \mathbf{q} \cdot \mathbf{r})=1$ for an ideal bcc crystal. Six reciprocal vectors with modulus equal to $2 \sqrt{2} \pi / a$ ( $a$ is the lattice constant) were used. The vectors $\mathbf{r}$ are the vectors between the center of mass of a molecule and each of its $Z$ nearest neighbors. In the calculation this local order parameter is further averaged over its neighbors. The order parameter so defined should be unity for a molecule in an ideal crystal and take on a small value for a liquid molecule. The order parameters of all the molecules of a typical configuration are shown in Fig. 1. We employ a cutoff value of $\phi_{c}=0.12$ to distinguish between crystal $\left(\phi>\phi_{c}\right)$ and melt $\left(\phi<\phi_{c}\right)$ molecules. Shown in the inset of Fig. 1 are the height functions for each of the two interfaces in the same configuration.

The values of the stiffnesses for each of the three slab orientations are obtained from the intercepts of plots of $\ln \left\langle|\widetilde{h}(k)|^{2}\right\rangle$ versus $\ln (k)$, shown in Fig. 2. The lines are weighted fittings to Eq. (2). The stiffnesses obtained by these fittings together with the free energies are shown in Table II. The orientationally averaged free energy is $\gamma_{0}=(7.0 \pm 0.4)$ $\times 10^{-3} \mathrm{~J} \mathrm{~m}^{-2}$. The anisotropy parameters are $\epsilon_{1}=(1.2 \pm 2.0)$ 


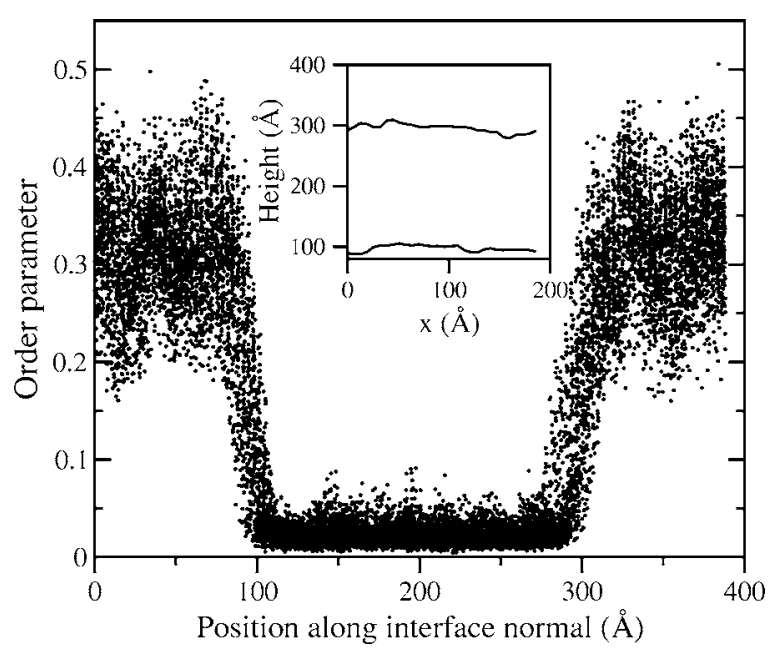

FIG. 1. The averaged order parameters for all molecules of a snapshot. The inset shows the interface heights for the same snapshot.

$\times 10^{-2}$ and $\epsilon_{2}=(1.4 \pm 4.6) \times 10^{-3}$. The results show that $\gamma_{100}$ $>\gamma_{110} \approx \gamma_{111}$, but the errors in the interfacial free energy and other related quantities are larger than the differences. The errors could be reduced further by longer runs, but given the small anisotropy in this system, it would require runs that are at least ten times longer simply to determine the signs of $\epsilon_{1}$ and $\epsilon_{2}$. Although the interfacial free energies themselves have large errors, the errors in the differences between $\gamma$ for different orientations are smaller. From the values of $\epsilon_{1}, \epsilon_{2}$, and $\gamma_{0}$, we have

$$
\begin{aligned}
& \gamma_{100}-\gamma_{110}=(5.8 \pm 8.6) \times 10^{-5} \mathrm{~J} \mathrm{~m}^{-2}, \\
& \gamma_{100}-\gamma_{111}=(5.3 \pm 9.5) \times 10^{-5} \mathrm{~J} \mathrm{~m}^{2}, \\
& \gamma_{110}-\gamma_{111}=(-1 \pm 7) \times 10^{-5} \mathrm{~J} \mathrm{~m}^{-2} .
\end{aligned}
$$

The orientationally averaged interfacial free energy $\left(\gamma_{0}\right)$ for succinonitrile determined from our simulation is about $20 \%$ lower than the experimental result of Schaefer et al. ${ }^{29}$ $\left[(8.9 \pm 0.5) \times 10^{-3} \mathrm{~J} \mathrm{~m}^{-2}\right]$, but within the error bars of the

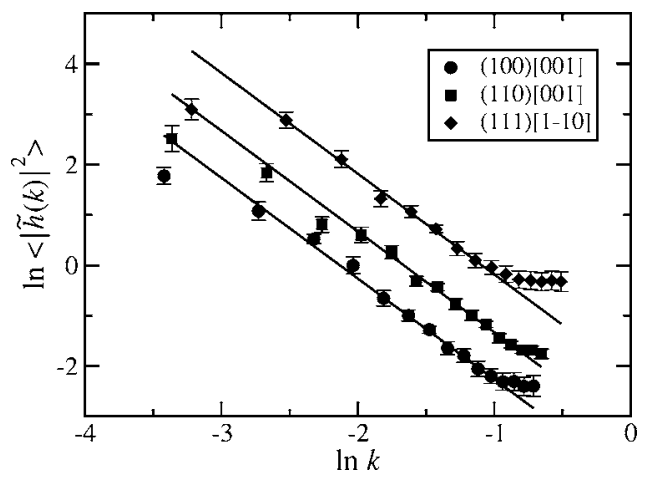

FIG. 2. The fluctuation spectrum of the quasi-one-dimensional interface height. The solid lines, which have slopes of -2 , are fits to the simulation results. The solid circles, squares, and diamonds are for (100)[001], (110)[001], and (111)[1피 interfaces, respectively. The indices in the parentheses indicate interface Miller indices, and the indices in the square brackets denote the "short" directions of the simulation box, which is of slab geometry. To be clear, the (110)[001] data have been moved along $Y$ axis by 1 , and (111)[1ํㅣ moved by 2 .
TABLE II. The interfacial stiffness $(\widetilde{\gamma})$ and free energy $(\gamma)$ for each interfacial orientation studied. $\tilde{\gamma}$ and $\gamma$ are in the unit of $10^{-3} \mathrm{~J} \mathrm{~m}^{-2}$.

\begin{tabular}{cccc}
\hline \hline Interface & Short direction & $\tilde{\gamma}$ & $\gamma$ \\
\hline$(100)$ & {$[001]$} & $6.60 \pm 0.7$ & $7.06 \pm 0.4$ \\
$(110)$ & {$[001]$} & $7.47 \pm 0.5$ & $7.00 \pm 0.4$ \\
$(111)$ & {$[1 \overline{1} 0]$} & $7.02 \pm 0.8$ & $7.01 \pm 0.4$ \\
\hline \hline
\end{tabular}

more recent value of $\left[(7.9 \pm 0.8) \times 10^{-3} \mathrm{~J} \mathrm{~m}^{-2}\right]$ of Maraşli et $a .^{30}$ However, even given the large experimental uncertainty, it is reasonable to conclude that our value is at least $10 \%$ lower than the true experimental value. Our simulation indicates that the anisotropy in the interfacial free energy is very small. The nearly isotropic character of SCN interfacial free energy is also observed in the experiments. To make contact with the experiments we determine the anisotropy coefficient $\epsilon_{4}$. Using Eq. (1), $\epsilon_{4}$ can be expressed in terms of $\tilde{\gamma}$ differences,

$$
\epsilon_{4}=\frac{\tilde{\gamma}_{(110)[001]}-\tilde{\gamma}_{(100)[001]}}{15\left(\widetilde{\gamma}_{(110)[001]}+\widetilde{\gamma}_{(100)[001]}\right)} .
$$

Using our values we obtain $\epsilon_{4}=(0.4 \pm 0.6) \%$, which is within the error bars of the values obtained by both Muschol et al. ${ }^{10}$ $[(0.55 \pm 0.15) \%]$ and Glicksman and Singh $^{9}(0.5 \%)$.

Using interfacial free energies obtained indirectly from nucleation rate experiments, Turnbull reported an empirical correlation between the interfacial free energy per surface particle $\hat{\gamma}=\rho^{-2 / 3} \gamma$ (where $\rho$ is the number density of the crystal) and the latent heat of fusion, $\Delta_{\text {fus }} H$,

$$
\hat{\gamma}=C_{T} \Delta_{\text {fus }} H / N_{A},
$$

where $N_{A}$ is Avogadro's number and $C_{T}$ the Turnbull coefficient, which was found by Turnbull to be about 0.45 for most metals and 0.32 for semimetals and water. For succinonitrile, the experimental crystal density and latent heat are determined to be $1.0158 \mathrm{~g} \mathrm{~cm}^{-3}$ and $3.704 \mathrm{~kJ} \mathrm{~mol}^{-1}$, respectively. ${ }^{39}$ Using these values, $C_{T}$ for $\mathrm{SCN}$ is found to be $0.33 \pm 0.03$ from the results of Maraşli et al. ${ }^{30}$ and $0.38 \pm 0.02$ from the results of Schaefer et al. ${ }^{29}$ The six-site model for succinonitrile gives $1.0108 \pm 0.0008 \mathrm{~g} \mathrm{~cm}^{-3}$ for the equilibrium crystal density and a latent heat of $3.11 \pm 0.19 \mathrm{~kJ} \mathrm{~mol}^{-1}$, which results in a $C_{T}$ of $0.35 \pm 0.02$. So the value of the Turnbull coefficient from the simulation is in agreement (within the error bars) with both experimental measurements, as well as being close to Turnbull's predictions for semimetals and water (0.32). The good agreement of our results for the Turnbull coefficient is due largely to the fact that both the latent heat and interfacial free energy are underestimated in the six-site model, and, because the Turnbull coefficient is a ratio of these two quantities, a partial cancellation of errors occurs. It is possible that an improved force field, with a more accurate latent heat, might also produce better interfacial free energies. Recently, Hoyt et al. ${ }^{40}$ reported values of the Turnbull coefficient of 0.29 from fluctuation method simulations on a variety of bcc metals (Fe, V, and Mo). In contrast, Turnbull coefficients for bcc soft-sphere systems were found, using the interfacial free energies from the cleaving method, to be larger $(0.45) .{ }^{19}$ These and the current 
results indicate that, unlike fcc materials, the Turnbull coefficients of bcc materials depend rather sensitively on the potential.

\section{CONCLUSION}

Using the capillary fluctuation method ${ }^{18}$ and a six-site molecular force field, ${ }^{34}$ we have calculated the crystal-melt interfacial free energy, $\gamma$, and its anisotropy for the model bcc-forming material succinonitrile. The magnitude of $\gamma$ is found to be about $20 \%$ lower than the experimental value obtained by Schaefer et al. ${ }^{29}$ but within the error bars of the more recent experimental result of Maraşli et al. ${ }^{30}$ The interfacial free energy is found to be very nearly isotropic, in agreement with experiment. ${ }^{9,10}$ In addition, the Turnbull coefficient for succinonitrile from our simulation $(0.35 \pm 0.02)$ is in agreement with the experiments within the error bars.

These results show that the capillary fluctuation method can be used to accurately determinate crystal-melt interfacial free energies for molecular systems.

\section{ACKNOWLEDGMENTS}

The authors wish to thank Dr. Ruslan Davidchack for helpful discussions. In addition, this research effort benefited greatly from the atmosphere of cooperation and collaboration provided by the Computational Materials Science Network program sponsored by the Department of Energy. For the funding of this work, we gratefully acknowledge support from the National Science Foundation under Grant No. CHE0316127.

${ }^{1}$ W. A. Tiller, The Science of Crystallization: Microscopic Interfacial Phenomena (Cambridge University Press, New York, 1991).

${ }^{2}$ I. V. Markov, Crystal Growth for Beginners: Fundamentals of Nucleation, Crystal Growth, and Epitaxy (World Scientific, Singapore, 1995).

${ }^{3}$ A. Karma and W.-J. Rappel, Phys. Rev. E 57, 4323 (1997).

${ }^{4}$ W. J. Boettinger, S. R. Coriell, A. L. Greer, A. Karma, W. Kurz, M. Rappaz, and R. Trivedi, Acta Mater. 48, 43 (2000).

${ }^{5}$ K. F. Kelton, Solid State Phys. 45, 75 (1991).
${ }^{6}$ L. Granasy and T. Pusztai, J. Chem. Phys. 117, 11121 (2002).

${ }^{7}$ S. Auer and D. Frenkel, Annu. Rev. Phys. Chem. 55, 333 (2004).

${ }^{8}$ J. M. Howe, Interfaces in Materials (Wiley, New York, 1997).

${ }^{9}$ M. E. Glicksman and N. B. Singh, J. Cryst. Growth 98, 277 (1989).

${ }^{10}$ M. Muschol, D. Liu, and H. Z. Cummins, Phys. Rev. A 46, 1038 (1992).

${ }^{11}$ K. Koo, R. Ananth, and W. N. Gill, Phys. Rev. A 44, 3782 (1991).

12 A. Dougherty and J. P. Gollub, Phys. Rev. A 38, 3043 (1988).

${ }^{13}$ P. Oswald, J. Phys. (France) 49, 1083 (1988).

${ }^{14}$ D. P. Woodruff, The Solid-Liquid Interface (Cambridge University Press, London, 1973).

${ }^{15}$ D. Turnbull, J. Appl. Phys. 21, 1022 (1950).

${ }^{16}$ J. Q. Broughton and G. H. Gilmer, J. Chem. Phys. 84, 5759 (1986).

${ }^{17}$ R. L. Davidchack and B. B. Laird, Phys. Rev. Lett. 85, 4751 (2000).

${ }^{18}$ J. J. Hoyt, M. Asta, and A. Karma, Phys. Rev. Lett. 86, 5530 (2001).

${ }^{19}$ R. L. Davidchack and B. B. Laird, Phys. Rev. Lett. 94, 086102 (2005)

${ }^{20}$ R. L. Davidchack and B. B. Laird, J. Phys. Chem. 118, 7657 (2003).

${ }^{21}$ J. Morris and X. Song, J. Chem. Phys. 119, 3920 (2003).

${ }^{22}$ J. Morris, Phys. Rev. B 66, 144104 (2002).

${ }^{23}$ D. Y. Sun, M. Asta, J. J. Hoyt, M. I. Medelev, and D. J. Srolovitz, Phys. Rev. B 69, 020102(R) (2004).

${ }^{24}$ M. Asta, J. J. Hoyt, and A. Karma, Phys. Rev. B 66, 100101(R) (2002).

${ }^{25}$ J. N. Sherwood, The Plastically Crystalline State: Orientationally Disordered Crystals (Wiley, New York, 1979).

${ }^{26}$ M. A. Eshelman and R. Trivedi, Acta Metall. 35, 2443 (1987).

${ }^{27}$ M. A. Chopra, M. E. Glicksman, and N. B. Singh, Metall. Trans. A 19, 3087 (1988).

${ }^{28}$ M. E. Glicksman, M. B. Koss, and E. A. Winsa, Phys. Rev. Lett. 73, 573 (1994).

${ }^{29}$ R. J. Schaefer, M. E. Glicksman, and J. D. Ayers, Philos. Mag. 32, 725 (1975).

${ }^{30}$ N. Maraşli, K. Keşlioğlu, and B. Arslan, J. Cryst. Growth 247, 613 (2003).

${ }^{31}$ B. B. Laird and R. L. Davidchack, J. Phys. Chem. B 109, 17802 (2005).

${ }^{32}$ J. J. Hoyt and M. Asta, Phys. Rev. B 65, 214106 (2002).

${ }^{33}$ W. R. Fehlner and S. H. Vosko, Can. J. Phys. 54, 2159 (1976).

${ }^{34}$ X. Feng and B. B. Laird, Mol. Phys. 103, 2795 (2005).

${ }^{35}$ B. B. Laird, J. Chem. Phys. 115, 2889 (2001).

${ }^{36}$ G. Cardini, R. Righini, and S. Califano, J. Chem. Phys. 95, 679 (1991).

${ }^{37}$ P. Derollez, J. Lefebvre, M. Descamps, W. Press, and H. Fontaine, J. Phys.: Condens. Matter 2, 6893 (1990).

${ }^{38}$ W. Smith, M. Leslie, and T. R. Forester, The DLPOLY_2 user manual, 2.14 edition, CCLRC, Daresbury Laboratory, Daresbury, England, 2003.

${ }^{39}$ C. A. Wulff and E. F. Westrum, Jr., J. Phys. Chem. Solids 67, 2376 (1963).

${ }^{40}$ J. J. Hoyt, M. Asta, T. Haxhimali, A. Karma, R. E. Napolitano, R. Trivedi, B. B. Laird, and J. R. Morris, Mater. Res. Bull. 29, 935 (2004). 